\title{
A Three Beam Approach to TEM Preparation Using In-Situ Low Voltage Argon Ion Final Milling in A FIB-SEM Instrument
}

\author{
E. L. Principe, P. Gnauck, P. Hoffrogge \\ Carl Zeiss SMT, Inc. Nanotechnology Systems Division \\ 555 Twin Dolphin Drive, Suite 130, Redwood City California, 94065 \\ Email: principe@smt.zeiss.com
}

Transmission electron microscopy sample preparation using focused ion beam instrumentation is now a well-established method suited for a broad range of materials. The popularity of the technique is due to advantages that include site specificity (while sacrificing a large viewable area) and the ability to automate milling of the membrane to electron transparency. FIB prepped samples have been shown to be of sufficient quality to generate "lattice" TEM images and it is also possible to physically thin a membrane to $30 \mathrm{~nm}$ or less, even using a $30 \mathrm{kV} \mathrm{Ga}^{+}$ion beam. However, optimal sample preparation requirements, for both TEM and STEM high-resolution imaging and analysis, demand that the membrane is not only thin; the membrane must also be uniform, have a minimal surface roughness and a minimal amorphous damage layer thickness ${ }^{1}$. Electron transparent membranes produced by typical FIB methods result in gallium implantation and also result in an amorphous layer, up to $20 \mathrm{~nm}$ in thickness. The amount of gallium implantation can be minimized through appropriate technique and amorphous damage layer thickness can be reduced by lowering the $\mathrm{Ga}^{+}$ion beam energy (i.e., $3-5 \mathrm{kV}$ ) during final thinning. The amount of gallium contamination and amorphous damage layer thickness that can be tolerated will ultimately depend upon the data requirements. Regardless, minimizing contamination of active elements and reducing the amorphous damage layer in an electron transparent membrane benefits the quality and accuracy of the high resolution microscopy and chemical analysis. It is interesting to note that these factors are even more relevant with the increasing popularity of high-resolution lowvoltage STEM, as even a small amorphous layer can reduce image contrast and result in beam broadening. Hybrid methods combine FIB membrane preparation with another final thinning process to improve the sample quality, such as chemical thinning or post ion milling. The latter approach typically involves ex-situ transfer of the sample to a low energy (i.e., $40 \mathrm{eV}-2 \mathrm{kV}$ ) ion mill.

This work will describe progress in the next logical step, incorporating a low energy ion gun within a FIB-SEM instrument to allow in-situ final milling of TEM membranes. In addition, an end-point detection scheme will be described that takes advantage of the ability to simultaneously monitor the membrane thickness with an independent electron beam, using multiple electron detectors. Aside from this key objective, other potentially interesting opportunities will be discussed that result from augmenting a FIB-SEM with a second low energy ion gun; the TripleCrossBeam ${ }^{\mathrm{TM}}$ concept.

\footnotetext{
1 "Thin Dielectric Film Thickness Determination by Advanced Transmission Electron Microscopy", A.C. Diebold, B. Foran, C. Kisielowski, D.A. Muller, S.J. Pennycook, E. L. Principe, S. Stemmer; Microscopy \& Microanalysis, 9, pgs 493-508, (2003).
} 